\title{
Networked experiments and scientific resource sharing in cooperative knowledge spaces
}

\author{
Sabine Cikic, Sabina Jeschke, Nadine Ludwig, Uwe Sinha and \\ Christian Thomsen
}

Technische Universität Berlin, MuLF-Center,

Faculty of Mathematics \& Natural Sciences, 10623 Berlin, Germany

Email: \{cikic,sabina.jeschke,nludwig,sinha,thomsen\}@math.tu-berlin.de

\begin{abstract}
Cooperative knowledge spaces create new potentials for the experimental fields in natural sciences and engineering because they enhance the accessibility of experimental setups through virtual laboratories and remote technology, opening them for collaborative and distributed usage. A concept for extending existing virtual knowledge spaces for the means of the technological disciplines ("ViCToR-Spaces" - Virtual Cooperation in Teaching and Research for Mathematics, Natural Sciences and Engineering) is presented. The integration of networked virtual laboratories and remote experiments ("NanoLab Approach"), as well as an approach to community-driven content sharing and content development within virtual knowledge spaces (NanoWiki) are described.
\end{abstract}

Keywords: Cooperative knowledge spaces, virtual laboratories, remote laboratories, wikis, nanotechnology

\section{INTRODUCTION}

Focusing on the social aspects of communication, coordination and cooperation, cooperative knowledge spaces possess a high potential to support the learning, teaching and research processes at universities by the means of new media and new technologies. "ViCToR-Spaces", currently under development at Technische Universität Berlin, focus on the enhancement of virtual cooperation in teaching and research in the fields of mathematics, natural sciences and engineering presenting novel collaborative working environments for knowledge acquisition and research as well as supporting natural forms of scientific and technological cooperations.
Cooperative knowledge spaces use a generalized "room metaphor" (Greenberg \& Roseman, 2003) as a guideline. They provide a virtual meeting point where interaction, communication and collaboration take place. The environment as a whole is defined by the combination of its dynamically linked objects, i. e. members, documents, tools and services (Hampel \& Keil-Slawik, 200I). Hence, the design of those components and the way they are linked is the key issue to successful cooperation and knowledge acquisition.

E-learning and e-research environments can be categorized into two main types: Within content-oriented system architectures, the content defines the center point of the system design, communicative and 
cooperative scenarios are (missing or) developed around the content objects. In community-oriented system architectures, communication and cooperation processes, as well as workflows between the users are the main focus of the system design while content objects are embedded into the cooperation infrastructure. As of now, content-oriented systems present the more "common" approach, forming the basis of most e-learning platforms currently available. Community-oriented systems can be regarded as part of the research field of CSCW/CSCL (Computer-supported Cooperative Work/Learning, (Borghoff \& Schlichter, 2000; Stahl, 2006)). Within this approach, social processes, represented by communication, coordination and cooperation, form the basis for successful knowledge acquisition in education as well as in research. Since this view is becoming increasingly accepted for computer-supported models of education and scientific work, community-oriented systems are currently under intense development.

Experiments play a vital role in natural and engineering sciences, thus their presence in form of virtual and remote laboratories in a cooperative knowledge environment is strongly desirable. We will have a closer look at cooperatively performed experiments in section 6, explaining why they are so essential to ViCToR-Spaces.

\section{RELATED WORK}

A wide variety of CSCW and CSCL environments has been developed during the last 15 years and many noteworthy results have been achieved.

Generic cooperative knowledge space platforms following the room metaphor include opens Team (Hampel \& Keil-Slawik, 2OOI) and CURE (Haake et al., 2004). opensTeam and CURE likewise are based on the concept of virtual rooms which serve as shared workspaces for user groups and as meeting places for students and teachers. Both platforms support shared viewing, exchanging and re-arranging of items, links between them, and discussions between users. In both platforms, new items may be created either by upload or using a built-in wiki engine. Moreover, both opens Team and CURE facilitate spontaneous forming of groups by providing awareness information and by managing access permissions on rooms and documents. Both platforms are being developed actively and are available under an open source license. Since presently CURE adheres more closely towards established standards and already provides a basic support for mathematical notations throughout its components, it is used as a technological basis to implement the first prototype of the ViCToR-Spaces concept (cf. section 4).

The Virtual Math Teams (Wessner et al., 2006) project (VMT) addresses virtual cooperation in the field of mathematics. Its most outstanding component is the ConcertChat system which combines a (virtual) shared whiteboard and a chat. These synchronous forms of communication and cooperation are in the main focus of the VMT project. Asynchronous cooperation, however, lies outside the scope of VMT proper; rather, it is one objective of the MathForum project which VMT is embedded into. Then again, MathForum only supports ASCII-based circumscriptions of mathematical formulae, i.e. " $\mathrm{x}^{\wedge} 2$ " for $\mathrm{x}^{2}$ etc.

Thus, the specific requirements for cooperative knowledge spaces for mathematics and natural sciences (Cikic et al., 2006) are not - or only rudimentally - supported by the systems presented in this section. This is probably the main reason for why there are still no satisfactory developments promoting community building and virtual cooperation in those disciplines.

\section{THE CONCEPT OF VIRTUAL ROOMS}

E-learning is most commonly associated with terms like Learning Management System (LMS) or Learning Content Management System (LCMS). In the last years, these types of learning systems have become widely accepted in educational institutions and companies. They are based on the concept of file systems in a computer. These systems provide a structure of documents, a history, and primarily a suggested thread through the offered material. Additionally, they provide communication interfaces like chats, discussion forums and email. These systems associate content-based learning with communication and sometimes even game-like concepts.

However, in "real life" learning as well as in research scenarios communication and cooperation play a most important role. Students form work groups to solve scientific problems or perform hands-on experiments together. Cooperation is also an important aspect in scientific collaboration of universities and other research institutions. Scientists of different institutions try to consolidate their knowledge for high-grade achievements in their research areas.

This fact leads to a change of paradigms. In addition to content-based systems, community-based systems are developed. Simple document management is enhanced to cooperative work in virtual 

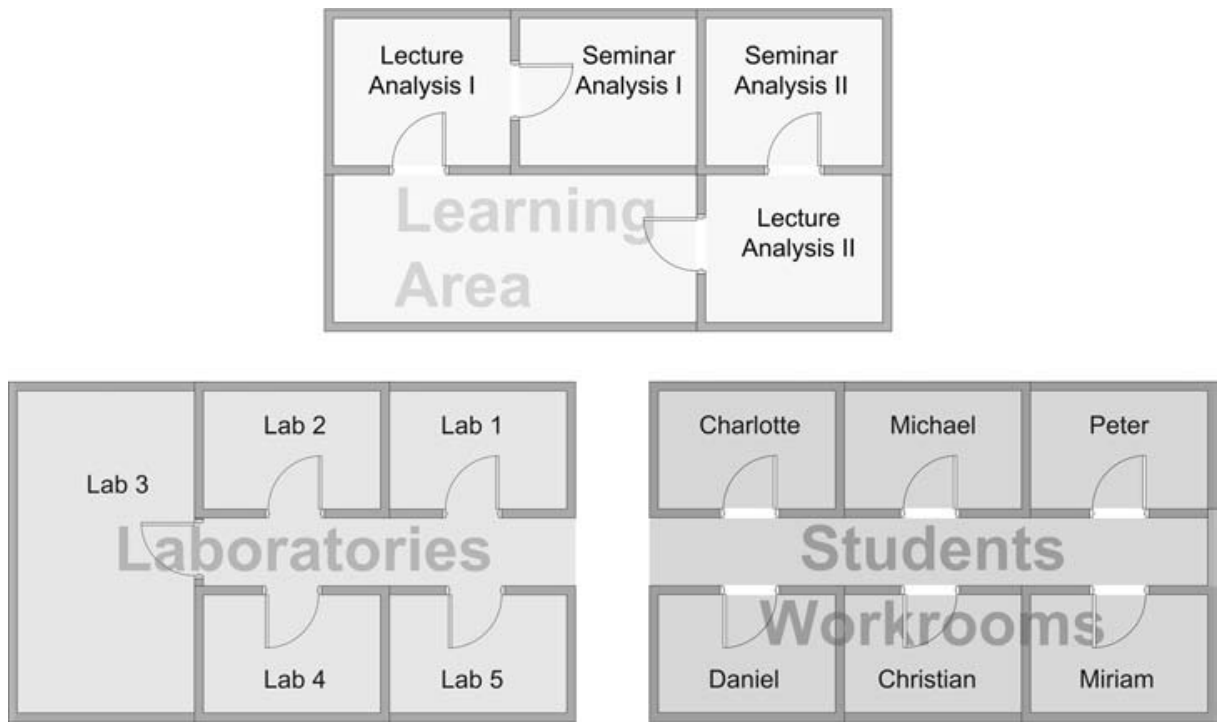

Figure 1 Different functional rooms (schema).

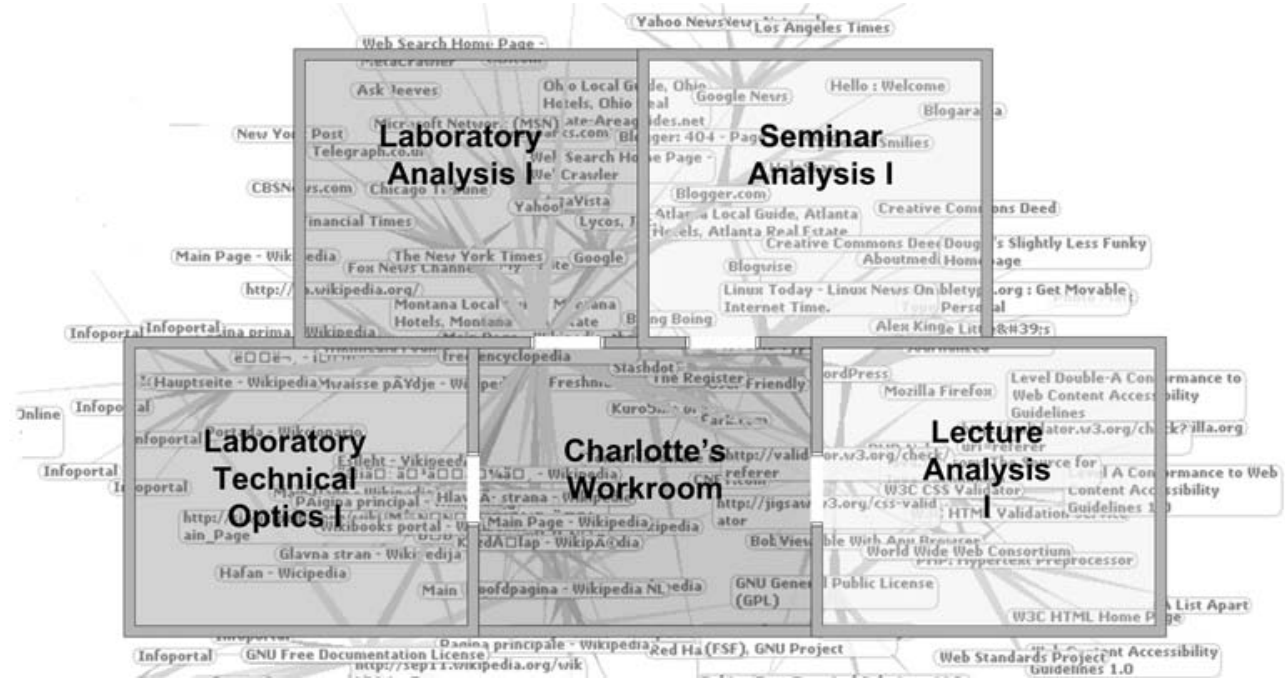

Figure 2 Rhizome-like arrangement of rooms.

rooms. This community-based style of learning is reflected in cooperative knowledge spaces. In the present article we introduce our room concepts for cooperative knowledge spaces.

Most community-based systems follow a roombased approach as it supports synchronous as well as asynchronous and mixed scenarios of cooperative learning (Wessner, 2005). These systems are composed of multiple rooms offering different functions. The function of a room is derived from the number and character of the room's users, the tools attached and the rights assigned in the room. Different combinations of these components form different types of rooms.

Following this principle, it is possible to design and create rooms for work groups, rooms with teaching material or laboratories with special virtu- al equipment according to the respective requirements. The users of such a system can decide on their own if they want to work in cooperation with other users or if they just want to solve tasks and study in their own workrooms. In addition, there is the possibility to build rooms as a mandatory element of a lecture series. Students will have to pass through some of the rooms (e. g. a laboratory) on their way through a seminar before the successful completion of the lecture series is accepted.

Special function rooms such as a room solely for teaching material or for a laboratory provide a clearly arranged structure of learning units offered in contrast to rooms containing all material of a complex lecture series. For example, a course consisting of a lecture, a seminar and an experiment can be distributed over three different rooms, which are 
presented in sequence or in parallel.

The function of a room will be constructed from three components already mentioned above:

- room-related rights

- specification of the room users

- tools and communication interfaces

By assembling rooms with different functions in sequence or in parallel (Figure I), a whole virtual university with lecture, seminar, workshop or laboratory rooms containing virtual and remote labs can be built up. By this means, a user can be virtually supported in all phases of a course, scientists from different institutions and locations can discuss their research achievements and approaches or perform experiments in virtual and remote laboratories - all in one virtual building.

The ViCToR-Spaces room concept does not provide the rooms in a hierarchical structure as it is characteristic for cooperative knowledge spaces currently deployed (cf. sec. 2). We are not designing a static root-to-leaf structure, but rather a dynamic, heterogeneous network, known in philosophy as a rhizome (Deleuze \& Guattari, 1987). Thus each user will obtain a different view on the room structure of the system according to the rooms which the user is interested in and related to. See Figure 2 for an example of the wikipedia network overlaid with the rhizome-like arrangement of the rooms from a user's perspective.

Users can be assigned to certain rooms supporting their individual profile, e. g. to the rooms of a course a student is attending in the current semester. Additionally, users can delete and add rooms constructing their individual network of rooms. The different perspectives of the users provide many different views on the room structure. The rooms define a new way of knowledge management.

Room-related rights

Virtual rooms, unlike physical rooms, can be built and deleted in short time. Obviously, not all users can be allowed to change or delete every room in the system. This makes rights management at room level necessary. Room-related rights have two different aspects. On the one hand, they refer to creating, deleting and altering a room. On the other hand, they define preconditions for accessing the room. For rooms that can or should only be used by a limited number of users, the access can be controlled by bookable time slots. Every user is assigned to limited time slots to conduct a hands-on experiment in a laboratory.

Another precondition for accessing a room, a special test room can be added upstream so that users first have to successfully complete the test before being allowed into the next room. This principle, i.e. the serial operation of completing a test, booking a time slot and finally conducting the hands-on experiment, deliberately introduces an artificial bottleneck. This is especially important in cases of special laboratories where users need previous knowledge for accomplishing a certain experiment.

\section{Specification of room users}

In most "real-life" seminars, three or four students form a work group to solve the tasks of the seminar jointly. In this case the room should "know" the identity of the group members to restrict access to the room solely to this user group. All participants have equal rights in the room concerning the modification of the room. A users' own workroom is another example of such a user-restricted room. Only the user himself can access his own workroom.

There are also rooms that should only be accessed by a fixed number of users, e. g. remote laboratories. In this case only the number of users who have access to the room is limited without specifying certain users.

A third possible type of room user specification is a room accessible to all users.

\section{Tools and communication interfaces}

As a third function component, a room can be filled in advance with tools and communication interfaces, according to the room function.

- Communication interfaces are important elements in virtual, cooperative work. In the ViCToRSpaces room concept we provide chats, discussion forums, email and a VoIP function. Not all interfaces need to be attached to all types of rooms. Small groups can manage a clear information exchange in a chat or VoIP session. In larger groups, however, such communication sessions can become unmanageable. In turn, smaller groups might not require a discussion forum.

- Shared whiteboards provide a common graphical work area for all room users. All participants are able to work with the same set of objects on the board. Similarly to a chat client, the written and drawn objects are shown identically on all participants' boards. Shared Whiteboards will be described in detail in section 4.

- Virtual and remote labs are mainly used in education for engineering and natural sciences. In both laboratories parameters can be manipulated, controlled and read out. Different measurement instruments can be attached and 


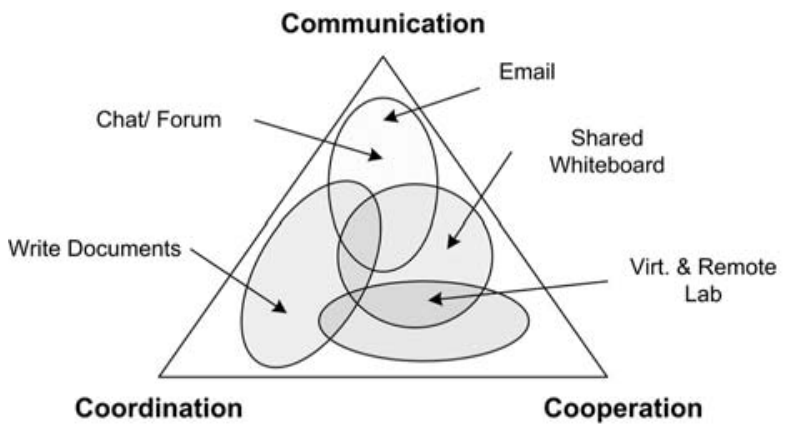

Figure 3 The " $3 \mathrm{C}$ model."

combinations of virtual and remote labs for the same physical effect can exchange parameters and other information. The concept of virtual and remote experiments will be outlined in section 5 .

The user in a virtual room

Within cooperative systems in general, the users' activities are characterized by the phenomena of communication, coordination, and cooperation the so-called "three C's":

- Coordination: In virtual knowledge spaces a student can get support in all phases of scientific or educational work. Students can form groups and make appointments for joint work sessions or hands-on experiments. Also, lecturers and students can meet in project rooms to resolve questions etc. The coordination in virtual knowledge spaces also brings scientists from different institutions together and supports their work.

- Communication interfaces are provided in multiple types. Students will mainly exchange information about a special lecture or difficult tasks. Lecturers will inform their students about news and the progress of a course and scientists will exchange research achievements or arising problems.

- Cooperation constitutes the essential element of virtual knowledge spaces. Students and scientists cooperate in sessions of hands-on experiments. They describe mathematical problems on shared whiteboards and discuss a physical algorithm over VoIP. In joint sessions they merge their knowledge and achieve high-grade research results. These achievements can be recorded in version-controlled documents if more than one session is needed.

The " $3 \mathrm{C}$ model" (Borghoff \& Schlichter, 2000) resulting from this view can be used to classify cooperative systems according to their support for each of these three phenomena. Figure 3 illustrates this for each component of a virtual knowledge space. The options a user has in a virtual knowledge space depend on the functionalities of the room. A group's workroom can be equipped with chat, a discussion forum, VoIP function and a shared whiteboard so that the users in this room can choose the type of communication best suited to solving their tasks.

\section{VICTOR-SPACES - COOPERATIVE ENVIRONMENTS FOR NATURAL SCIENCES}

ViCToR-Spaces focus on community-oriented eLTR-technologies (e-learning, e-teaching and eresearch) in the fields of mathematics and natural sciences. In order to avoid "reinventing the wheel", ViCToR-Spaces are built on top of the CURE platform briefly described in section 2 .

Since the requirements for cooperative knowledge spaces for mathematics and natural sciences differ greatly from what is supported by existing solutions, there still is a lack of implementations (Cikic et al., 2006). Integration of virtual labs and remote experiments and the realization of experiments conducted cooperatively form one of the most important aspects and are described in section 5 in detail. Another important aspect in ViCToR-Spaces is the principle of cooperative design, storage and reuse of content. This principle is implemented exemplarily for nanoscience in the NanoWiki approach which will be outlined in section 7 .

In the following, we will discuss additional, special requirements for $\mathrm{ViCToR}-S p a c e s$ briefly. These requirements result from the daily practice of students, teachers, researchers and users of mathematics and natural sciences.

Support of special notations (e. g. mathematical or chemical formulae) within all tools When exchanging mathematical knowledge in a web-based CSCL or CSCW system, one of the problems most likely encountered is the limited support for writing anything other than plain text. Special notations such as mathematical formulae (where, besides special characters, fractions, sums, integrals, superscripts, subscripts etc. are needed) or diagrams (e. g. UML) cannot be readily entered into forums, wikis or chat systems. These tools have to be extended to support MathML which has been developed for the professional presentation of mathematical formulae at websites.

Authoring tools with MathML support, based on LaTeX, incl. WYSIWYG front-ends The editing process for formulae encoded in 
MathML still represents a complex task which has to be supported by suitable authoring environments. These editing tools should be based on common standards such as LaTeX because of being well-accepted among mathematicians and users of mathematics, and also because of the variety of existing LaTeX-to-Math-ML converters. However, since competence in LaTeX is far from universal, especially among students, an integrated graphical tool (WYSIWYG front-end) for editing mathematical formulae is an important extension for interdisciplinary cooperation within virtual knowledge spaces.

\section{Distributed field-specific editing on shared whiteboards}

The traditional way of cooperation between mathematicians or natural scientists uses face-to-face communication, where two or more scientists discuss a formula, develop a proof, draw a schematic diagram for an experiment together on a chalkboard or a whiteboard, each of them writing their annotations between their co-researcher's writings. This way, of course, requires each of the participants to be at the same place at the same time. Geographical distances and various other reasons, however, make this an option which is not always viable.

Shared whiteboards represent an approach to overcome these distances. Generally speaking, shared whiteboards serve as a common workspace for all members of a group - e. g. the aforementioned mathematicians developing a proof cooperatively. Thus, all members of that group can work on the same set of data synchronously. The whiteboard itself is a virtual or real drawing board - e.g. E-Chalk (Friedland et al., 2004) - on which objects are displayed graphically and can be manipulated. Very much like a chat client, the data which users are working on is displayed identically on each user's whiteboard.

For both synchronous and asynchronous distributed cooperative writing, some sort of rights management is essential. Lack of rights management may cause content to be changed in a way contradictory to the original creator's ideas, or may allow malevolent users to join a shared whiteboard session and arbitrarily change or even delete objects on the board. Likewise, both forms of distributed cooperative writing require a suitable version management which allows tracing back changes and, if necessary, reverting a content item or a whiteboard session to an earlier state.

Integrating existing eContent from various repositories

Plenty of e-learning content (eContent) material has been developed in the fields of mathematics, natural sciences and engineering, mostly in previous projects on a local or national level, driven by individual researchers and their institutes. This material is not restricted to text-based knowledge elements, but includes a large amount of multimedia objects and interactive components instead. So far, these resources are widely spread, stored in local databases of e-learning and e-research projects only accessible to small, restricted user communities. In the meantime, however, the development of appropriate metadata formats and other standardization efforts have provided the means to build large, comprehensive knowledge repositories.

\section{Information Retrieval}

Since such content repositories mentioned above consist of a huge number of elements, automated information retrieval systems capable of handling text, sound, images, data and other objects are becoming more important. So far, web search engines such as Google are the most visible applications of research on information retrieval. To handle scientific and educational material, advanced search mechanisms are needed which are capable of interpreting the content of an object in a more comprehensive manner. Semantically enhanced information retrieval techniques are being developed which are usually based on computer linguistic analysis combined with statistical methods.

Apart from the aforementioned field-specific demands, current implementations of virtual knowledge spaces suffer from a "lost in cyberspace phenomenon", which has to be addressed by continuative actions towards user support. This includes enhanced transparency of the structure of the virtual space, further development of awareness components for community support, the trans-

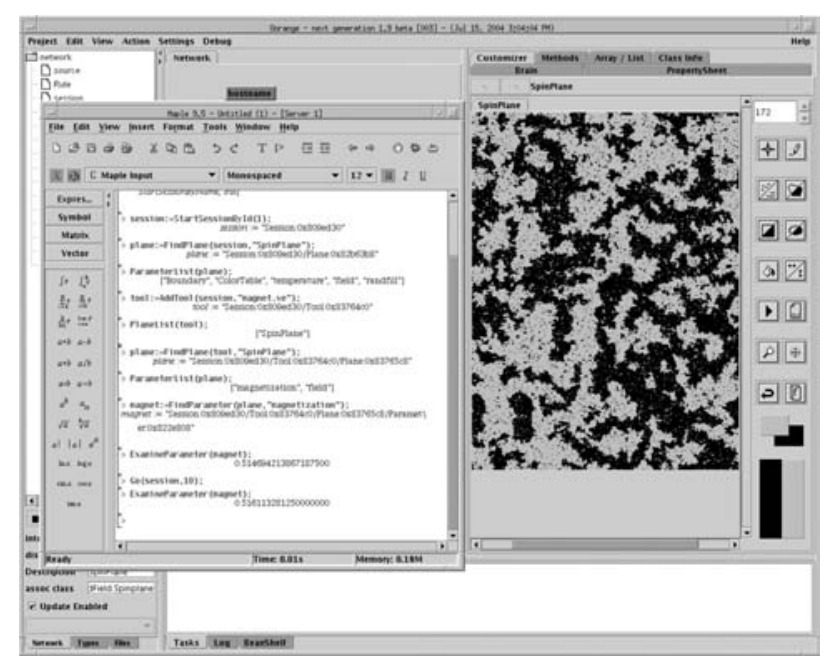

Figure 4 Screenshot of the VideoEasel 


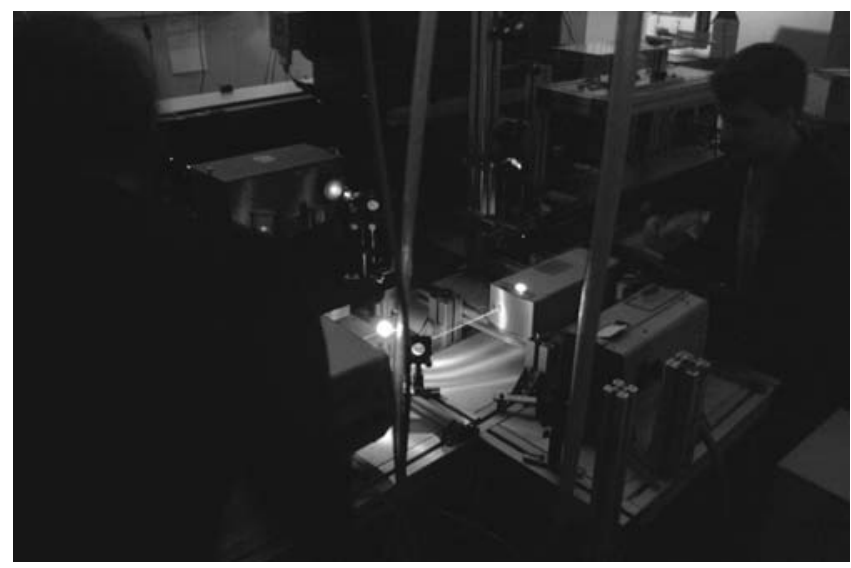

Figure 5 The remote laser in the lab....

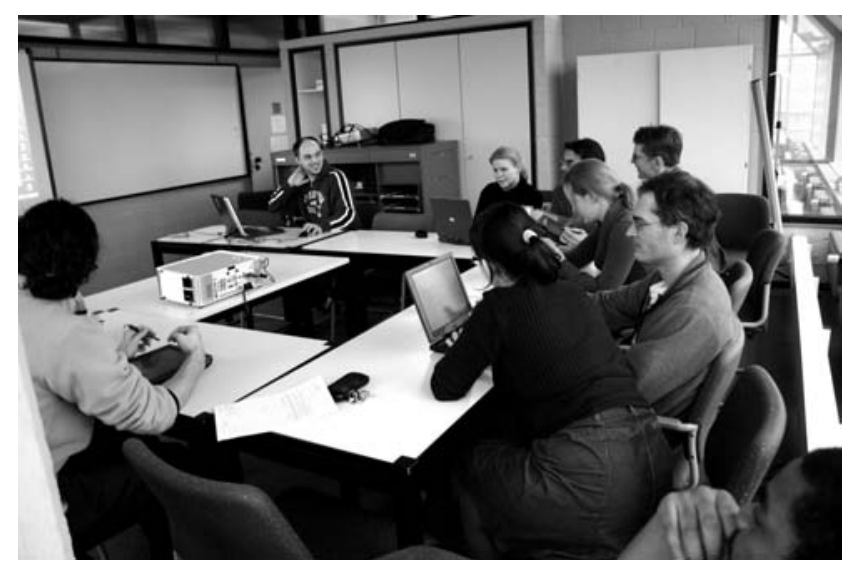

Figure 6: $\ldots$ and the experimenters outside the lab, using Tablet-PCs to perform experiments

parency of workflows for different types of actions, transparency of content management through ontologies and intelligent semantic retrieval mechanisms. Possible solutions to these questions will be discussed in a subsequent paper.

\section{EXPERIMENTS IN VIRTUAL SPACES}

Experiments form an important part of learning, teaching and research within the technological disciplines. Integrated into a cooperative knowledge space, they provide better access to experimental setups for all students, independent of limitations in time, budget or access to classical laboratories thus forming one of the most important parts of the ViCToR-Spaces concept.

To enhance access to experimental setups, there are two principle alternatives (Jeschke et al., 2005b): Virtual laboratories and remote experiments form the basis for the NanoLab-project. Virtual laboratories use the metaphor of a "real" scientific laboratory as a guiding line (Jeschke et al., 2005a; Jeschke $\&$ Richter, 2006). The software design focuses on emulating scientific hands-on experience in virtual spaces (see Figure 4 for an example). In theoretical fields such as mathematics and theoretical physics, virtual laboratories have revolutionized education and research as they allow an intensive experimental access to abstract objects and concepts. They are capable of building bridges between the theoretical fields and experimental sciences.

Complementary to virtual laboratories, remote experiments are real experiments, remotely controlled by the experimenter from outside the laboratory (Thomsen et al., 2005). They are based on a technology which allows true experimenting from a remote location at almost any given time; this is illustrated in Figures 5 and 6 . The different approaches have a number of similarities, and enrich each other through their differences: remote experiments allow the investigation of real objects including hands-on measurement experience, which does obviously not hold for virtual laboratories. On the other hand, virtual laboratories are capable of constructing an experiment, whereas this kind of flexibility is hard to imagine or implement in remote experiments.

\section{THE NANOLAB APPROACH}

Nanoscience and nanotechnology have a tremendous impact on current technological development and are therefore considered to be key technologies of the 2ist century. New discoveries and developments in physics, electrical engineering as well as in life sciences show the advance of objects of nanometers in size. In contrast to the current submicron technology, the major impact of nanotechnologies is not based on the small size itself. Rather, the reduced size and dimensionality results in a wealth of new physical properties, which make nanoscience increasingly responsible for a farreaching technological paradigm change.

This development has only become possible because of the increased sophistication in preparation and experiment and is paralleled by the enormous advancement of accurate calculational tools. The steadily rising computational power and the shrinking system sizes have nearly converged to a device size where structural, electronic and vibrational properties can - and have to be - computed with full quantum mechanical complexity. Carbon nanotubes or semiconductor quantum dots are examples where anisotropy and reduced dimensionality play an additional important role in understanding and exploiting nano-systems.

The use of technology-enhanced learning and research methods and tools is crucial to support the 
resulting paradigm change and to initiate rapid knowledge transfer and state-of-the-art education. In particular, since the experimental and theoretical techniques in nanosciences are extremely complex and require expensive equipment, e.g. clean rooms, atomic-force microscopes or highly sophisticated spectrometers, facilities for experiments in nanoscience are restricted to a rather small community. Additionally, extensive numerical techniques such as molecular dynamics simulations or timedependent ab initio calculations as theoretical tools are now commonly used in nanosciences and nanotechnologies to predict or optimize physical properties. Considerable cost and experience is necessary to employ these techniques successfully and efficiently.

This results in a bottleneck for teaching, research and development in nanotechnologies since the required systems and facilities are not available everywhere. Future effectiveness in nanotechnological education and research - and future economic growth - strongly depend on the ability to provide its researchers and developers with extended access to the most advanced generation of e-research facilities for nanoscience. Thus, nanoscience and nanotechnology require a consolidated and cooperative effort for their successful implementation in future technology and production processes. Here, new forms of web-based collaboration between academic institutions will be one of the central keys for success in nanoscience and nanotechnology.

Designing and implementing a service-oriented infrastructure, targeting on distributed collaborative composition and execution of experiments in natural sciences including data analysis, interpretation of the results, and development of applications is the overall objective of the NanoLab approach. A common portal infrastructure will enable access to virtual and remote experiments in Europe through standardized interfaces. In NanoLab, experiments (regardless of whether they are remote experiments on real physical devices or virtual experiments that are simulated or combinations of both) can be seen as complex processes that consist of various technical and non-technical components (devices, sensors, effectors, analytical components, software services, humans etc.). These components need to be orchestrated into a complex system to perform an experiment. In general, such systems might be distributed. Components of experiments are reusable within other experiments and exchange happens even across different scientific communities that are working on the NanoLab platform. The NanoLab faces a severe heterogeneity challenge: a community-driven NanoLab has to enable different providers to design and implement single elements which can be combined with already existing tools within an experiment. Thus, ensuring interoperability across the platform is a non-trivial but very important task. The set of available components for experiments has to be dynamic: new components will be invented by providers and advertized within the platform whereas other components that might be outdated or (in the case of physical devices) temporarily not available will be removed from the platform. Thus, the NanoLab platform can be regarded as an open marketplace for experimental components.

The organization of all cooperative processes plays an important role within the NanoLab concept. A scenario of collaborative work involving instantaneously connected mobile users in virtual knowledge spaces is structured according to the three phases: Formation (establishing the network infrastructure and group structure), collaboration (structuring and organizing knowledge), and closure (retaining the results). Collaborative work in knowledge spaces connotes creating, sharing, and structuring of results and documents in various ways. Collaborative composition of documents in a Wikistyle manner (sec. 7) can be as much part of the collaboration as can the usage of whiteboards or any other mode of synchronous and asynchronous cooperation. The collaboration phase of an ad-hoc group is characterized by the exchange and structuring of materials within the mobile knowledge space. For this purpose the attendees insert documents into the knowledge space to share them with the group. The collaboration may happen synchronously by using a shared whiteboard presenting a shared view to the knowledge space. Similar to scientific practice in a laboratory, the group members can cooperatively explore interactive experiments placed in the knowledge space and develop formal representations of the experiments.

The change from synchronous to asynchronous cooperative work in mobile collaboration scenarios is often seamless. While face-to-face cooperation is the natural form of collaboration in mobile scenarios, the participants may leave the collaboration session. Thus, mobile forms of virtual knowledge organisation provide additional challenges to CSCW systems. Classical, centralized collaboration systems have always provided their service via a dedicated server. In the novel distributed collaboration systems, services are provided by several nodes of a peer-to-peer network with all participants being mobile. Small or larger groups establish ad-hoc collaboration networks. Meanwhile users join existing networks for some period of time and then leave 
again. The classical separation of service provider (server) and service consumer (client) dissolves. The technical terminus "peer" becomes a terminus for a special form of collaboration by sharing collaboration services and resources with each other.

Interconnectedness of the different laboratories and experiments and their embedding into the ViCToR-Spaces is not to be restricted to the purely technical level of IT-integration, but has to include networking on the content level through field-specific ontologies, Semantic Web technology and innovative models of dynamic semantic process composition. Thus, the ViCToR-Spaces have to provide a virtual environment in which collaborative efforts like knowledge distribution and cooperative experiments in nanoscience will be managed intelligently. Semantic Web technologies form the basis to achieve these goals: The Semantic Web aims at augmenting the existing World Wide Web with machine-readable semantics, making the content of today's Web accessible to intelligent queries and machine reasoning. Taking a more abstract perspective, Semantic Web is concerned with the semantically meaningful and well-defined description of abstract resources, for instance documents, graphics, data streams etc. which allow machines to access and deal with abstract resources. In contrast to standard (i.e. not semantically annotated) resources, semantically annotated resources can be integrated automatically and processed dynamically (without deep and detailed prior agreements between the providers of different resources). Effectively, this means that such resources can be integrated across time and communities in a flexible way. This is particularly important for open systems that have many contributors from various communities and systems that change their structure and configuration at runtime. The NanoLab platform as envisioned in this concept is a perfect example of such a system.

\section{THE NANOWIKI APPROACH}

The "Nano Spaces" described in section 6 have to contain learning and teaching material and background descriptions of the different experiments as well as access to digital libraries and other scientific resources. Plenty of eContent material has been developed in the fields of nanoscience and nanotechnologies, mostly in previous projects on a local or on a national level, driven by individual researchers and their institutes. This material is not restricted to text-based knowledge elements, but rather includes a high degree of multimedia objects and interactive components. Combining and reusing these manifold resources enable the design and implementation of high-quality course material, interactive self-tests, examinations as well as integration of experimental settings and data.

Through their very unique, unconventional and powerful way of web-based collaboration, Wikis (Cunningham \& Leuf, 200I) have revolutionized traditional notions of cooperation and knowledge exchange. The NanoWiki concept provides novel collaborative authoring environments for knowledge storage and dissemination, accessible through Web Services. In traditional content management systems a restricted author group develops content modules, subsequently releasing them to the user community. In contrast, Wiki environments empower each user to become a contributor ("open editing"), thus already simplifying access to a broad variety of repositories. The Wiki approach does not only help generating new types of content archives - it also induces new user-driven procedures and work sequences as well as new forms of community development.

The central goal of the NanoWiki approach is to provide a basic collection of existing, distributed "eNanoContent" (eContent for nanosciences) in order to build a comprehensive knowledge repository. Based on the Wiki concept, NanoWiki realizes an open "lightweight" architecture for community-driven content sharing and content development in nanoscience. Semantic content encoding as well as the extended use of metadata enables intelligent forms of content retrieval and navigational structures adequate for complex, interdisciplinary, fast-growing sciences. New ontologies help to organize the knowledge based on quality criteria and thus provide reusability in different communities or for different applications, such as teaching in high schools, universities, or company training. Thus, NanoWiki will both enrich nano-related eContent and multiply eContent accessibility.

The following main challenges have to be addressed:

- Content structuring \& semantical content enrichment: To meet the demands of a self-growing network, innovative knowledge systems need to arrange their content according to field-specific ontologies including semantically well-defined metadata and semantic encoding of the scientific content. These features are not implemented in current Wiki engines, searching and retrieval methods are mainly restricted to title and full text search, interconnections between different content objects are invariably realized through simple links. Due to the complexity of the task, only a small number of indi- 
viduals has been able to participate in the process of ontology building in the past. Recently, the innovative OntoWiki technology has suggested how to incorporate ontology development throughout an entire user community (Hepp et al., 2005).

- Quality control: The need for quality control in a rapidly growing Wiki environment is the subject of current debate. If intended for educational use, however, quality control is a prerequisite to guarantee scientific excellence and international acceptance. Within NanoWiki a peerreview system will be integrated, ensuring the content quality through a board of internationally recognized internal and external experts. Part of the challenge is the proper balance between quality and speed of content growth.

- Representing/editing contents in natural science: Existing Wiki implementations have to be extended by additional features essential for the appropriate presentation of mathematical and natural science related content. This includes the conversion of the defacto-standard LaTeX into XML/MathML, support of handwriting recognition technologies (specifically adapted to mathematical and chemical formulae), scribble tools to add simple sketches, charts, diagrams and experimental setups as well as connections to virtual laboratories and remote experiments.

- Knowledge representation and user adaptivity: The aforementioned ontological structures form the basis for the realisation of non-linear navigation structures, the integration of conceptual graphs and storyboard concepts for individual learning paths and automatically generated knowledge nets, converting traditional content management systems into explorative user-centric knowledge spaces.

To enhance access to digital sources, the NanoWiki approach will also make extended use of Semantic Web Technologies and Semantic Web Services: Ontologies - here in the sense of machine interpretable, shared conceptualisations of a domain are the backbone of the emerging Semantic Web. They define the concepts and entities of a domain of discourse, as well as their properties and the relations between them. Organized by ontologies and semantically described, resources can be integrated across time and communities in a flexible way. Considering the heterogeneous repositories mentioned above, a semantic description of the contents will significantly simplify the handling of contents from these various sources or types.
Thereby, semantic encoding of scientific content for different types of media, including appropriate metadata, are the basis of many next-generation system-components and demands as re-usability, interoperability, knowledge retrieval, and feedback handling on user input. In order to appropriately present mathematical and natural science related content (MathNatWiki), existing Wiki implementations have to be extended by a number of additional features. To deal with the complexity of digital content, ontologies come into place. Thereby, incorporation of ontology development throughout an entire user community (OntoWiki) is an important issue to meet the demands of a self-growing network, in particular in such an emerging and innovative field as nanoscience and nanotechnologies. Specific subject-matter ontologies enable the machine-processable description of the various entities which can be combined to complex units for heterogenous target groups. Furthermore, these ontologies will provide the structure for all new knowledge objects, annotations and changes of all documents that are created. Heterogeneities in the various documents and digital sources are unavoidable and will be faced by technical means for ontology mediation. Principled and machine-supported change management of documents will ensure consistency of the documents. Semantic Retrieval Environments can produce so called knowledgewebs or other logically organized presentations of text-based contents playing an important role to enhance the possibilities for students to choose their individual way of learning. Finally, the integration of appropriate composition and navigation tools is an important issue for the presentation of interconnections between the different terms, concepts and algorithms represented by the various knowledge objects.

\section{OUTLOOK}

In the past years, the main focus in developing eLTR-technologies has been on stand-alone applications and solutions for specific tasks. Today, modern approaches in the design of the architectures required show that the integration and interconnection of independent, single components play a central role in providing diverse, comprehensive functionality and addressing a broad, heterogeneous user spectrum.

As a result, we face two serious challenges: First, the next period in information technology will be dominated by demands for application integration. Research and applications are increasingly oriented 
towards semantic content encoding as a prerequisite for interconnectedness on a content level and towards integrative technologies for software components as "Web Services/Semantic Web". Second, integration on a social, community-oriented level, that is, support of communication and cooperation structures and shared workflows, is becoming more and more important. The human desire for communication and cooperation - as a basis for successful knowledge acquisition - has been largely deferred in favor of self-determined learning and work, independent of time and location. Not realizing that communication and cooperation in a virtual world will even facilitate one's need for mobile and freelanced access to knowledge we currently face a knowledge landscape of widely spread developments and unequally distributed knowledge.

Crosslinking existing knowledge repositories and developments will open knowledge and technologies to students, teachers and researchers beyond geographical limitations by advancing the building of virtual communities. Instancing ViCToR-Spaces combined with the NanoLab and NanoWiki approaches reveals the high potential of networked learning, working and researching. Democratic sharing in an open-source environment (where open-source is not only related to software developments but as an ideal for community building) will save precious time and money for the people, organizations and institutions involved and increases the value of all the numerous and great developments already made.

\section{REFERENCES}

Borghoff, U. M. and Schlichter, J. H. (2000). ComputerSupported Cooperative Work: Introduction to Distributed Applications. Springer, Berlin/Heidelberg.

Cikic, S., Jeschke, S. and Sinha, U. (2006). ViCToRSpaces: Cooperative knowledge spaces for mathematics and natural sciences. In Reeves, T. C. and Yamashita, S. F., editors, Proceedings of E-Learn 2006, pages 2358-2365, Chesapeake, AACE

Cunningham, W. and Leuf, B. (200I). The Wiki Way. Quick Collaboration on the Web. Addison-Wesley, Boston.

Deleuze, G. and Guattari, F. (1987). A Thousand Plateaus. University of Minnesota Press, Minneapolis.
Friedland, G., Knipping, L., Schulte, J. and Tapia, E. (2004). E-Chalk: a lecture recording system using the chalkboard metaphor. International fournal of Interactive Technology and Smart Education, I(I):9-20.

Greenberg, S. and Roseman, M. (2003). Using a room metaphor to ease transitions in groupware. In Ackerman, M., Pipek, V., and Wulf, V., editors, Sharing Expertise: Beyond Knowledge Management, pages 203-256. Cambridge, MA, MIT Press.

Haake, J. M., Schümmer, T., Haake, A., Bourimi, M. and Landgraf, B. (2004) Supporting flexible collaborative distance learning in the CURE platform. In Proceedings of the 37th Annual Hawaii Int. Conference on System Sciences, Los Alamitos, CA, USA. IEEE Computer Society.

Hampel, T. and Keil-Slawik, R. (200I) sTeam: Structuring information in a team - distributed knowledge management in cooperative learning environments. F. Educ. Resour. Comput., I(2es):3.

Hepp, M., Bachlechner, D. and Siorpaes, K. (October 2005) OntoWiki: Community-driven Ontology Engineering and Ontology Usage based on Wikis. Proceedings of the 2005 International Symposium on Wikis (WikiSym 2005), San Diego, California.

Jeschke, S. and Richter, T. (2006) Mathematics in virtual knowledge spaces - user adaptation by intelligent assistants. In Kaschek, R. and Jantke, K.-P., editors, Intelligent Assistant Systems. Idea Group, Hershey.

Jeschke, S., Richter, T. and Seiler, R. (2005a) VideoEasel: Architecture of virtual laboratories for mathematics and natural sciences. page 874 ff., Badajoz. FORMATEX.

Jeschke, S., Richter, T., Scheel, H., Seiler, R., and Thomsen, C. (September 2005b) The Experiment in E-learning: Magnetism in Virtual and Remote Experiments. Conf. Proc. ICL 2005, Interactive computer aided learning, Villach/Austria, Kassel/Germany. Kassel University Press.

Stahl, G. (2006) Group Cognition: Computer Support for Building Collaborative Knowledge. MIT Press, Cambridge (Mass.).

Thomsen, C., Scheel, H., and Morgner, S. (June 2005) Remote Experiments in Experimental Physics. Proceedings of the ISPRS E-Learning 2005, June I-3, Potsdam/Germany.

Wessner, M. (2005) Kontextuelle Kooperation in virtuellen Lernumgebungen, volume 8 of Schriften $z u$ Kooperations- und Mediensystemen. Josef Eul Verlag, Lohmar - Köln.

Wessner, M., Shumar, W., Stahl, G., Sarmiento, J., Mühlpfordt, M. and Weimar, S. (2006) Designing an online service for a math community. In ICLS 'o6: Proceedings of the 7 th international conference on Learning sciences, pages 818-824. Int. Society of the Learning Sciences 
Sabine Cikic holds a degree in Public and Private Environmental Management from Freie Universität Berlin. She has worked on the management of data on contaminated soils at the District Environment Office in Berlin-Kreuzberg and IUP Ingenieure GmbH. She is currently completing a degree in Geography, Geology, and Geophysics with a diploma thesis on Chinese migrants and food in Paris. In August 2005 Ms Cikic joined the MuLF team (Center for New Media in Education and Research). Her research field is focused on virtually spatial, interactive and distributed scenarios in e-learning and e-research.

Since 2005, Sabina Jeschke is associate professor for 'New Media in Mathematics and Natural Sciences' and director of the MuLF Center (Multimedia Center for New Media in Education and Research) at Berlin University of Technology (TU Berlin). The research of S. Jeschke is aimed at developing new concepts of service-oriented architectures for eLearning and eScience in particular in the fields of mathematics, natural sciences and engineering. She focuses on development and networking of interactive mathematical objects and remote/virtual experiments, on mechanisms for the creation, modification and storage of data in cooperative virtual knowledge spaces, and on the design of intelligent data analysis and validation schemes.

Nadine Ludwig graduated from Technische Universität Ilmenau with a degree in Computer Science in 2005. In her diploma thesis she described the integration of remote laboratories in Learning Content Management Systems via SCORM. Since May 2006 Ms Ludwig is a part of the MuLF Center at Technische Universität Berlin as a research assistant. Currently she is working on her PhD Thesis in the fields of eLearning and eResearch in Cooperative Knowledge Spaces.

Uwe Sinha holds a degree in Computer Science from Technische Universität Berlin. After graduation he worked for Berlin-based IVISTAR AG where he developed a web-based facility management system. In 2002 he joined the MUMIE project at Technische Universität Berlin. In that project a second-generation e-learning platform geared to mathematics was developed. Currently Mr Sinha is working on his $\mathrm{PhD}$ thesis with the research focus on computer-supported cooperative learning in mathematics, natural sciences and engineering.

Christian Thomsen, with a PhD from Brown University in 1986, has been a Professor of Physics at the Berlin University of Technology since 1994. He is a renowned scientist with over 380 publications in solid-state physics. His Berlin group has been a driving force behind the development of multimedia technologies at the university starting in 1996, implementing multimedia educational elements in the education of undergraduate students, in particular for engineering students. Christian Thomsen served the Berlin University of Technology as Vice President (1997-99) and as Dean of the Faculty of Mathematics and Natural Sciences (2003-). 